\title{
Fairness versus Efficiency
}

\author{
- An Experimental Study of (Mutual) Gift Giving -
}

\author{
Werner Güth, Hartmut Kliemt and Axel Ockenfels *
}

December 2000

\begin{abstract}
Fairness is a strong concern as shown by the robust results of dictator giving and ultimatum experiments. Efficiency, measured by the sum of individual payoffs, is another potential concern in games such as the prisoners' dilemma and public good provision games. In our experiment participants can increase efficiency by gift giving at the cost of reducing their own monetary payoff. In the one-sided treatment this is only possible for one of the two partners. The two-sided treatment allows for mutual gift giving. In both cases decisions can be conditioned on whether there is or there is not an efficiency gain by gift giving. The overall conclusion from our results is that striving for efficiency is constrained by equity concerns that are less stringent in mutual exchanges than in onesided gift-relationships.
\end{abstract}

\footnotetext{
* Güth: Humboldt-University of Berlin, Department of Economics, Institute for Economic Theory III, Spandauer Str. 1, D-10178 Berlin, Germany, +49-30-20935731, fax 49-30-20935704; gueth@wiwi.huberlin.de. Kliemt: Department of Philosophy, Gerhard Mercator-University, D-47048 Duisburg, Germany, +49-203-3792243, fax 49-203-3792243; Hartmut.Kliemt@t-online.de. Correspondence to: Ockenfels: Faculty of Economics and Management, University of Magdeburg, P.O. Box 4120, D-39016 Magdeburg, Germany; +49-391-6712197, fax+49-391-6712971; axel.ockenfels@ww.uni-magdeburg.de.
} 


\section{Introduction}

In dictator experiments two parties, $X$ and $Y$, can share a monetary pie $p$ of fixed size. $X$, the "dictator", makes the only decision $x$, with $0 \leq x \leq p$, of the underlying "dictator game" and thereby allocates monetary payoffs $p-x$ to $X$ and $x$ to $Y$, the "recipient". Neglecting other regarding preferences (see on utility interdependencies the classification in $\mathrm{Ng}, 1979$, chap. 1) $X$ can, in principle, consider two dimensions of value, his own payoff $p-x$ and the fairness of the payoff vector $(p-x, x)$. Depending on the size of $x$ the two considerations will be either in harmony or conflict. If $X$ regards more egalitarian distributions as more fair than less egalitarian ones then every choice of $x$ from the interval $(p / 2, p]$ is strictly dominated along both the personal gain and the fairness dimension in this range by $x=p / 2$. Over the range $(0, p / 2)$, on the other hand, any change in $x$ either increases $X^{\prime}$ 's personal gain at the expense of rendering the distribution less egalitarian or the fairness of the distribution is increased at the expense of a decreasing payoff $p-x$ for $X^{2}$

While in dictator experiments any final allocation is efficient, dictator dilemma experiments, as first introduced by Ockenfels (1999), create a sharp trade-off between fairness and efficiency. More specifically, in dictator dilemma games $Y$ receives more than what $X$ gives. Let $e(>0)$ denote $X$ 's monetary endowment. The choice $x$ with $0 \leq x \leq e$ allocates the monetary payoff $e-x$ to $X$ and the payoff $m x, m>1$, to $Y$. The larger $x$ the larger the payoff sum $e+(m-1) x$. A dictator $X$ who places positive value on the total payoff has good reason to consider also choices $x$ in the range $x>p / 2$. Therefore in the dictator dilemma, contrary to the dictator experiment, even over the range $x>e /(m+1)$ in which fairness could be increased by a reduction of $x$ such a reduction of $x$ is not necessarily the only plausible choice. Since in addition dictator dilemma games do not create incentives for strategically motivated other-regarding

\footnotetext{
${ }^{1}$ Bolton, Katok and Zwick (1998) study various experimental dictator games and review earlier dictator game studies in experimental economics. Related experiments were performed by social psychologists who first let participants work and then allocate their common reward $p$ knowing $X$ 's contribution to $p$; see, for instance, Shapiro (1975), and for a more recent study with entitlements by experimental economists Hoffman and Spitzer (1985).
} 
behavior, they are an appropriate tool to measure the relative impact of fairness and efficiency considerations.

The experiment whose result we describe and discuss subsequently combines aspects of the dictator and the dictator dilemma game. Like in the dictator game the player who has to make an actual decision can unilaterally allocate monetary amounts to himself and a recipient. What $X$ gives to $Y$ is either doubled, i.e. $m=2$, or just passed on, i.e. $m=1$. Doubling occurs with probability $5 / 6$ while the monetary amount is passed on with complementary probability of $1 / 6$. By allowing an $X$-participant to condition his gift $x$ to $Y$ on whether the gift is doubled (the gift $x_{2}$ ) or not (the gift $x_{1}$ ), we learn for every $X$ participant how she would behave in a dictator experiment and in a dictator dilemma experiment. Therefore, beyond Ockenfels (1999) "within"-subject comparisons are possible. $^{3}$

Two different treatments were used, both relying on the game sketched here.

In the one-sided treatment there are players in the dictator role and others who are serving as recipients only. None serves in both roles. The two-sided treatment introduces symmetry in the sense that not only $X$ chooses $x_{1}$ for $m_{x}=1$ and $x_{2}$ for $m_{x}=2$ but that also $Y$ makes corresponding decisions $y_{1}$ for $m_{y}=1$ and $y_{2}$ for $m_{y}=2$. Depending on the chance moves and their simultaneous decisions $x_{1}$ and $x_{2}$, on the one and $y_{1}$ and $y_{2}$ on the other hand, $X$ respectively $Y$ earn:

$$
\begin{array}{lll}
e-x_{1}+y_{1} ; e-y_{1}+x_{1} & \text { for } & m_{x}=m_{y}=1 \\
e-x_{2}+y_{1} ; e-y_{1}+2 x_{2} & \text { for } & m_{x}=2, m_{y}=1 \\
e-x_{1}+2 y_{2} ; e-y_{2}+x_{1} & \text { for } & m_{x}=1, m_{y}=2 \\
e-x_{2}+2 y_{2} ; e-y_{2}+2 x_{2} \text { for } & m_{x}=m_{y}=2
\end{array}
$$

\footnotetext{
${ }^{2}$ The theory of cognitive dissonance (Festinger 1964) suggests that participants might tend to avoid conflict through prioritizing either fairness, choosing $x=p / 2$, or personal gain, choosing $x=0$. In such cases the focus would be completely on one while leaving out of account the other concern completely. While this happens quite frequently, many other individuals are compromising their two values in the sense of choosing an $x$ from the range $0<x<p / 2$ where fairness and self-interest conflict.

3 One could argue that contrary to what rationality implies, $X$-participants will consider the overall stochastic choice problem. Since our data are in line with the results of Ockenfels (1999) who did not apply the strategy method, this argument seems lacking importance.
} 
Compared to the one-sided treatment, in the two-sided treatment gift giving can be supported by the hope that the partner will donate as well. Such expectations should lead to larger gifts, mainly in case of $m=2$ but also when $m=1$. Efficiency would require $x_{2}=y_{2}=e$. However, donating the full endowment requires trust in the other's willingness to act likewise if fairness is a critical consideration. The experiments provide some insights in what may happen in such situations.

In the following section 2 we describe the experimental procedure in some detail. Sections 3 and 4 present the results of the one-sided, respectively two-sided treatment. Final observations in section 5 conclude the paper.

\section{Experimental Procedure}

The experiments have been performed at the Humboldt University in Berlin. Participants were recruited from students attending an undergraduate course in microeconomics. 24 participants played the two-sided treatment (since both partners decide independently, this provides 24 independent observations). In the one-sided treatment we also had 24 pairs yielding 24 independent observations for 'dictators' $X$ and 24 observations for 'recipients' $Y$ (who were asked for hypothetical choices: "what would you give if you were $X$ instead of $Y$ ?").

Participants were urged to carefully read the instructions (the instructions are available from the authors upon request), then they received their decision forms with two control questions, checking whether the rules were understood, and asking for the (hypothetical) decisions. The final question elicits in an elementary way expectations about gift giving by others.

In the one-sided treatment three participants, one dictator and two recipients, did not answer both control questions correctly (see individual data file in the Appendix). In the more complex two-sided treatment also three participants (\# 3, 11, and 17) failed to understand the experiment fully. Leaving these participants in our data file would not have questioned our principal effects. In our analysis, however, we rely exclusively on the choices of subjects who answered the control questions correctly. 
An experimental session lasted about 30 minutes. The initial endowment was always $e=\mathrm{DM} 10$. We allowed for non-integer choices $x_{1}$ and $x_{2}$. The data file in the Appendix lists all 72 observations (including the six that are left out in the analysis since they came from participants who did not answer the control question correctly).

\section{One-sided gift giving}

The actual (hypothetical) rounded $x_{1}$ and $x_{2}$-choices by dictators (recipients) in the onesided treatment are graphically illustrated in Figures 1a (actual choice) and $1 \mathrm{~b}$ (hypothetical choices). Table 1 additionally provides summary statistics like the average relative gift $x_{1}^{r}=x_{1} / 10, x_{2}^{r}=x_{2} / 10$, or $x^{r}=\left(x_{1}+x_{2}\right) / 20$ and their respective standard deviations (first rows). Participants' responses to the question of what they expected as donations are represented, too, by expectation-averages and their standard deviations (second rows).

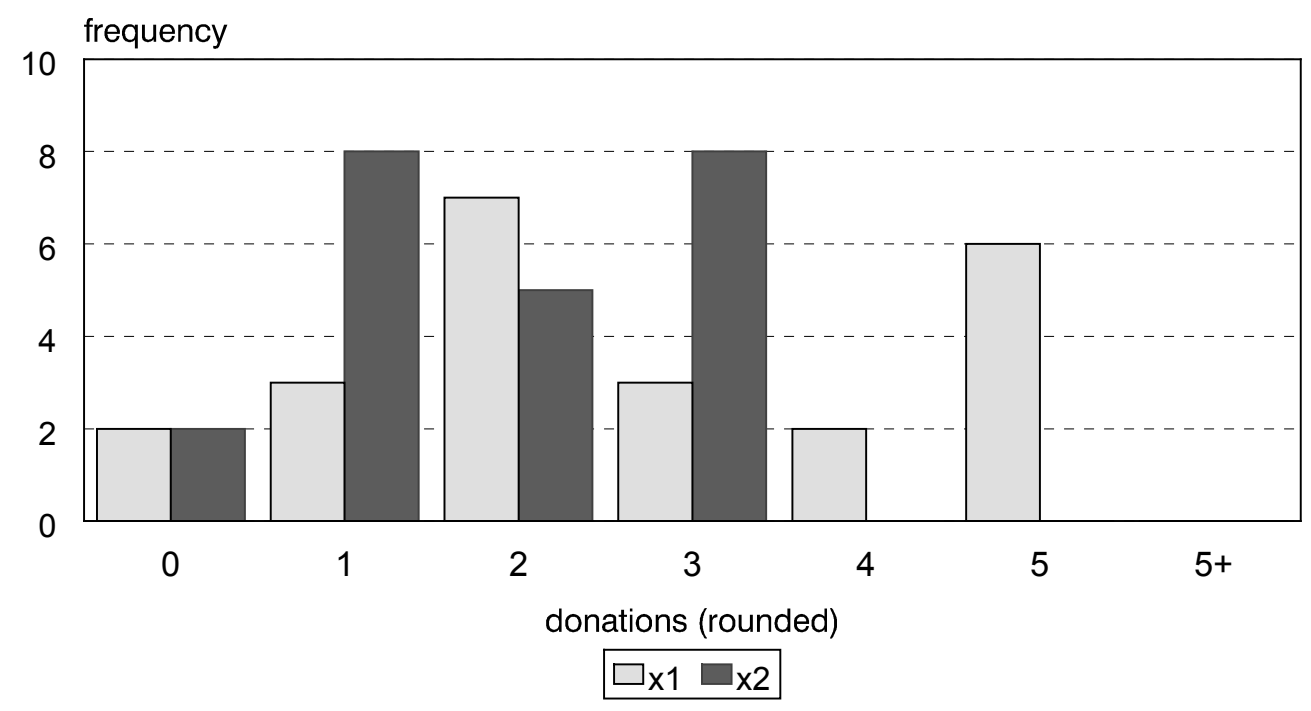

Figure 1a: Distribution of the donations of $X$ in the one-sided treatment 


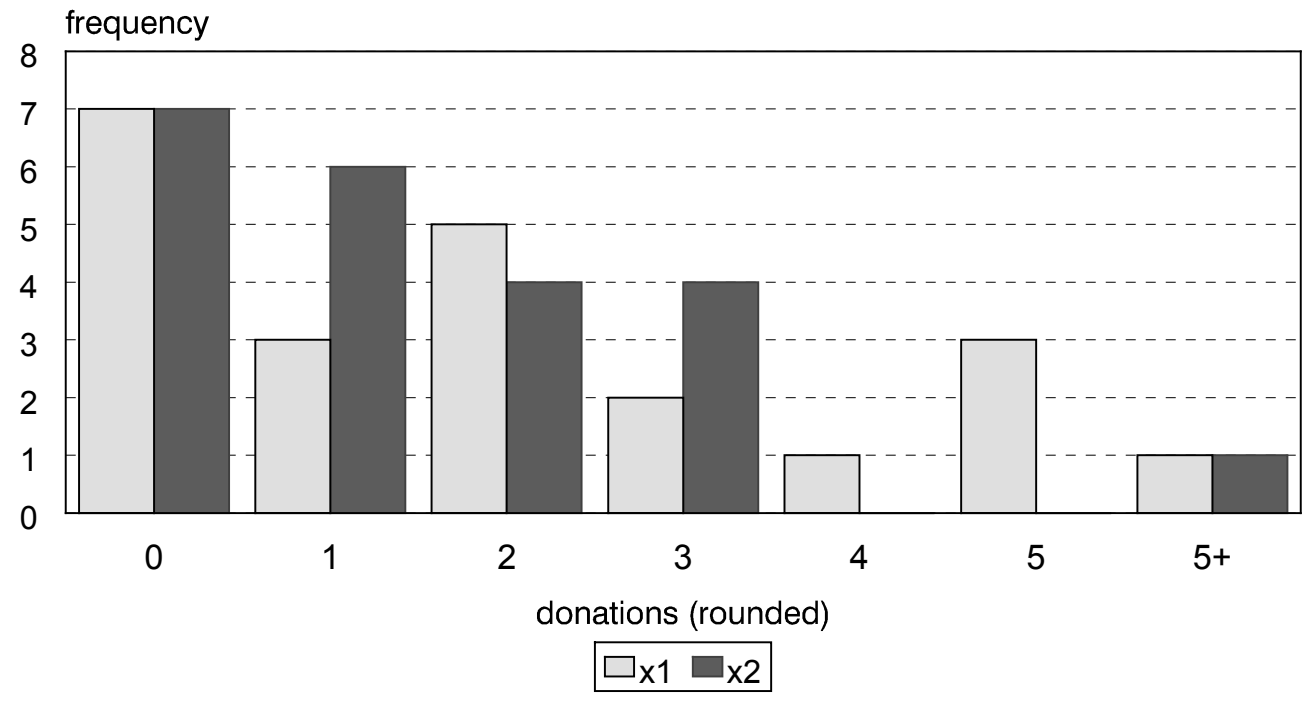

Figure 1b: Distribution of the hypothetical donations of $Y$ in the one sided treatment

\begin{tabular}{|c|c|c|c|c|c|}
\hline \multicolumn{2}{|c|}{ passing on } & \multicolumn{2}{|c|}{ doubling } & \multicolumn{2}{c|}{ both } \\
\hline$x_{1}^{r}$ & $\sigma_{x_{1}^{r}}$ & $x_{2}^{r}$ & $\sigma_{x_{2}^{r}}$ & $x^{r}=\frac{x_{1}+x_{2}}{20}$ & $\sigma_{x^{r}}$ \\
\hline
\end{tabular}

\begin{tabular}{|c|c|c|c|c|c|c|c|c|}
\hline \multirow{2}{*}{$\begin{array}{c}\text { one- } \\
\text { sided }\end{array}$} & $\mathrm{X}$ & chosen & 27.3 & 16.7 & 17.0 & 9.1 & 22.1 & 12.6 \\
\cline { 2 - 9 } & $N=23$ & expected & 27.0 & 13.9 & 16.7 & 9.8 & 21.8 & 11.5 \\
\cline { 2 - 9 } & $\mathrm{Y}$ & chosen & 21.6 & 24.8 & 15.9 & 21.7 & 18.8 & 22.8 \\
\cline { 2 - 9 } & $N=22$ & expected & 13.8 & 13.9 & 11.3 & 11.2 & 12.5 & 11.8 \\
\cline { 2 - 9 } & $\varnothing$ & chosen & 24.4 & 20.7 & 16.4 & 15.4 & 20.4 & 17.7 \\
\cline { 2 - 9 } & $N=45$ & expected & 20.4 & 13.9 & 14.0 & 10.5 & 17.2 & 11.6 \\
\hline
\end{tabular}

Table 1: Averages and standard deviations of relative donations of $X$ and $Y$ in the one-sided treatment (in percent)

Without exception $x_{1} \leq 5$ and $x_{2} \leq 10 / 3$ so that no $X$-participant ever granted a higher payoff to her $Y$-partner than to herself. This clearly confirms 
Regularity 1: In the one-sided treatment fairness concerns dominate efficiency considerations in the sense that dictators never put themselves at a relative disadvantage even if that be efficiency enhancing (for $m=2$ ).

In view of Regularity 1, one can speak of a one-sided fairness constraint in the sense of $x \leq e /(m-1)$ which makes sure that $X$-dictators never get less than their $Y$-partner (see also Ockenfels, 1999). This, of course, does not exclude payoff vectors yielding less to $Y$ partners, i.e. allocations brought about by a choice $x<e /(m-1)$. Actually, since the average $x_{1}^{r}$ is 27.3 and the average $2 x_{2}^{r}$ is $34\left(<83=100-x_{2}^{r}\right)$, the $Y$-partners earn about $1 / 3$ of what dictators get in case of $m=1$ and only a slightly higher share in case of $m=$ 2. As revealed by Table 1 this coincides pretty well with expectations. Since the average expectations of $X$-participants differ from average behavior only by $0.3 \%$ for $x_{1}$ and $x_{2}$, we can note

Regularity 2: Actual choices and expected choices of dictators $X$ are nearly identical.

Regularity 2 is based on averages and therefore would not in principle exclude the possibility that generous $X$-participants expect meager gifts and vice versa. This possibility is, however, ruled out by the strong positive correlation between $x_{1}$ and $x_{2}$ choices and their corresponding expectations for others (the highly significant Spearman rank correlation coefficients are 0.633 for $x_{1}$ and 0.753 for $x_{2}$ ). This justifies

Regularity 3: Generous donors expect generosity to prevail in general. ${ }^{4}$

The intra-personal comparison of individual $x_{1}$ - and $x_{2}$-choices may provide some clue as to what may have been motivational forces behind the observed patterns of choice behavior. Table 2 gives a complete classification of $\left(x_{1}, x_{2}\right)$-vectors as fixed by $X$ participants, namely

\footnotetext{
${ }^{4}$ A similar effect has been observed by Selten and Ockenfels (1998) in the context of the solidarity game.
} 
- the egoistic type with $x_{1}=x_{2}=0$,

- the constant-sacrifice type with $x_{1}=x_{2}>0$,

- the constant-gift type with $x_{1}=2 x_{2}>0$,

- the intermediate (of the two previous) types with $2 x_{2}>x_{1}>x_{2}>0$,

- $\quad$ and the efficiency-minded type with $x_{2}>x_{1} \geq 0$.

\begin{tabular}{|c|c|c|c|c|c|c|}
\hline \multicolumn{2}{|c|}{ types } & egoistic & \begin{tabular}{c} 
fixed total \\
sacrifice \\
\multicolumn{2}{|c|}{}
\end{tabular} & intermediate & $\begin{array}{c}\text { fixed gift to loser } \\
\text { (exact or } \\
\text { up to rounding) } \\
x_{1}=x_{2}=0\end{array}$ & $\begin{array}{c}\text { efficiency- } \\
\text { guided }\end{array}$ \\
\hline$X$ & $\#$ & 2 & 6 & $x_{1}=0$ & $2 x_{2}>x_{1}>x_{2}>0$ & $x_{2}>x_{1} \geq 0$ \\
& $\%$ & $9 \%$ & $26 \%$ & 3 & 12 & 0 \\
\hline$Y$ & $\#$ & 7 & 5 & 0 & $92 \%$ & $0 \%$ \\
& $\%$ & $32 \%$ & $23 \%$ & $0 \%$ & $41 \%$ & $5 \%$ \\
\hline
\end{tabular}

Table 2: Types of conditional donations of $X$ - and $Y$-players in the one-sided treatment

Among the individuals assigned the dictator role $X$ the major categories are the constant-gift type with 52\% and the constant-sacrifice type with (26\%). More than $90 \%$ of all $X$-participants engaged in gift-giving, i.e. were non-egoistic. The only zero-gifts came from the two egoistic types. No $X$-participant was efficiency-guided. We observe

Regularity 4: Selfish behavior in the sense of contributing zero-gifts is very rare, and is not shown even if unselfish behavior cannot affect efficiency gains.

Table 2 also includes hypothetical choices of recipients $Y$ who were asked to imagine that they were in fact acting in the dictator role. It is rather surprising that $Y$-participants in their imagined decision chose on average lower $x_{1}$ - and $x_{2}$-values. Since $Y$-choices are purely hypothetical, it would have been cheap (talk) to display generosity. Yet the differences between the distributions of actual and hypothetical choices are insignificant 
(two-sided Mann-Whitney $U$-test, $p=0.127$ and 0.186 for $x_{1}$ and $x_{2}$, respectively). However, $Y$-participants expect significantly lower $x_{1}$ - and $x_{2}$ - gifts by others $(p=0.003$ and 0.061 for $x_{1}$ and $x_{2}$, respectively) than $X$-participants. Expectations distinguish $X$ and $Y$-participants according to

Regularity 5: While hypothetical gifts are only slightly smaller than payoff-relevant gifts, recipients are substantially less optimistic about gift giving by others than dictators.

Like actual dictators most recipients (41\%) in their imagined choices as dictators are of the constant-gift type. But the second largest group are the egoistic types $(32 \%)$ although the constant-sacrifice type is also non-negligible $(23 \%)$. The efficiency-minded type is extremely rare (just one $Y$-participant).

\section{Mutual gift giving}

Figure 2 graphically illustrates the (rounded) $x_{1}$ - and $x_{2}$-choices observed in the twosided treatment. Due to the symmetry of mutual gift giving all participants encounter the same decision problem. To distinguish from the asymmetric roles of $X$ and $Y$ in the onesided treatment we refer to these participants as Z-players.

Comparing Figure 2 with Figures 1a and $1 \mathrm{~b}$ the distributions are much more spread out: ${ }^{5}$ Neither $x_{1}>5$ nor $x_{2}>10 / 3$ are excluded.

\footnotetext{
5 The standard deviations are smallest for actual dictators, intermediate for hypothetical dictators, and largest for mutual gift givers.
} 


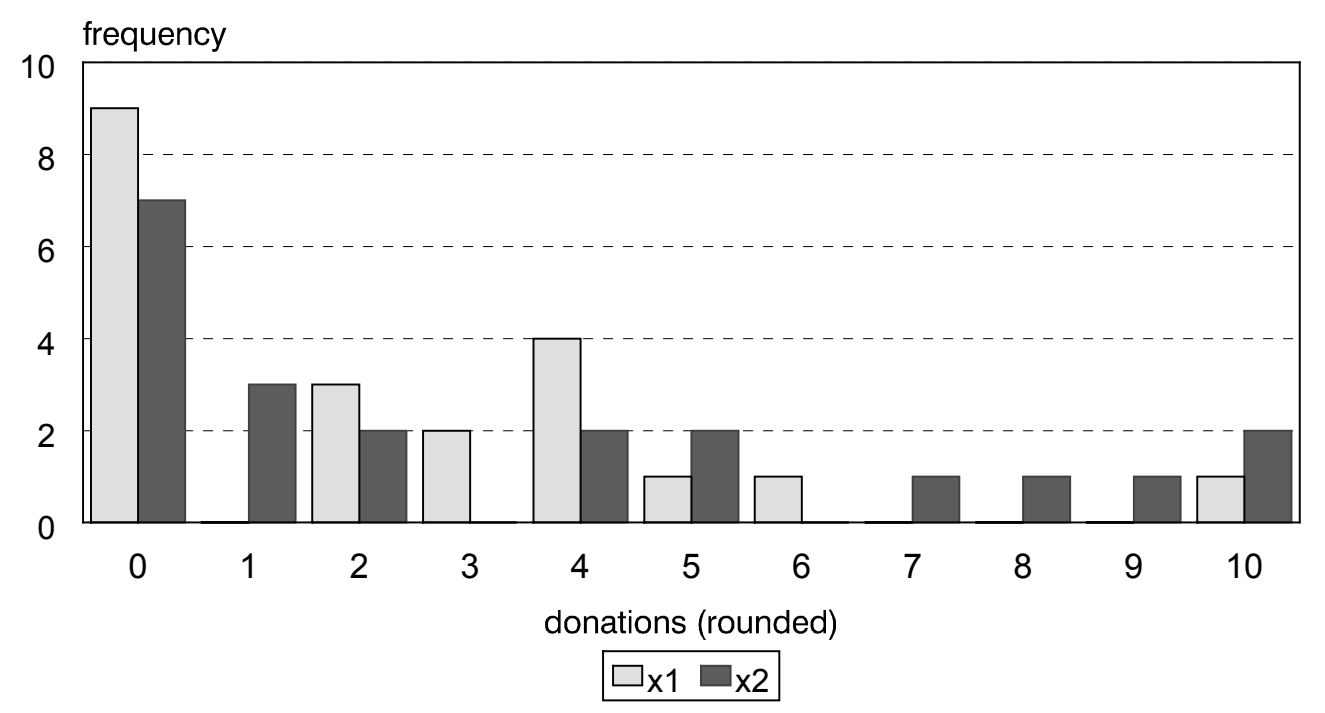

Figure 2: Distribution of the donations in the two-sided treatment

Table 3, which corresponds to Table 1, reveals that compared to unilateral dictators mutual gift givers choose slightly (but not significantly) lower $x_{1}$-gifts but significantly larger $x_{2}$-gifts (two-sided Mann-Whitney $U$-test, $p=0.052$ ). Most importantly, while in the one-sided treatment $x_{1}^{r}$ is substantially larger than $x_{2}^{r}$, both among dictators and among recipients, the opposite is true in case of mutual gift giving.

\begin{tabular}{|c|c|c|c|c|c|}
\hline \multicolumn{2}{|c|}{ passing on } & \multicolumn{2}{|c|}{ doubling } & \multicolumn{2}{|c|}{ both } \\
\hline$x_{1}^{r}$ & $\sigma_{x_{1}^{r}}$ & $x_{2}^{r}$ & $\sigma_{x_{2}^{r}}$ & $x^{r}=\frac{x_{1}+x_{2}}{20}$ & $\sigma_{x^{r}}$ \\
\hline
\end{tabular}

\begin{tabular}{|c|c|c|c|c|c|c|c|c|}
\hline $\begin{array}{c}\text { two- } \\
\text { sided }\end{array}$ & $Z$ & chosen & 23.1 & 26.5 & 33.0 & 36.0 & 28.0 & 28.2 \\
\hline
\end{tabular}

Table 3: Averages and standard deviations of relative donations of $Z$ in the two-sided treatment (in percent)

This justifies to state the following

Regularity 6: The possibility of receiving a donation from the recipient of one's own donation strengthens efficiency considerations for $m=2$ while leaving the proclivity to donate unaffected in the absence of efficiency gains; i.e. if $m=1$. 
Similar to Regularities 2 and 3, average expectations concerning gift giving by others are only insignificantly below actual average choices while expectations and own choices are highly significantly correlated (the Spearman rank correlation coefficients are 0.586 for $x_{1}$ and 0.600 for $x_{2}$ ).

Table 4 applies the same classification of types as in Table 2 to the two-sided treatment. Compared to the dictator dilemma case (52\%) the constant-gift type is quite rare $(14 \%)$ in case of mutual gift giving whereas the efficiency-minded type ranges rather prominently ( $23 \%$ instead of $0 \%)$.

It seems that mutual gift giving induces more extreme behavior than one-sided gift giving. At least not only the efficiency-minded type but also the egoistic type is more frequent in the two-sided than in the one-sided gift-relationship. The reasons for this may be that trust and uncertainty play a much stronger role in the case of two-sided gift giving than in case of unilateral donations. For the dictator uncertainty about the receiver is naturally absent and therefore such uncertainty cannot provide a reason or an excuse for non-generous behavior. In the shadow of uncertainty about the behavior of others in the case of mutual gift giving, however, individuals may feel that they are not acting egoistically if they do not contribute efficient amounts. What in the one-sided case would be unfair "greed" is now "protection" against exploitation (defensio in Hobbes' terminology of the De Cive, see also the English translation 1998).

\begin{tabular}{|c|c|c|c|c|c|c|}
\hline \multicolumn{2}{|c|}{ types } & egoistic & $\begin{array}{c}\text { fixed total } \\
\text { sacrifice }\end{array}$ & intermediate & $\begin{array}{c}\text { fixed gift to loser } \\
\text { (exact or } \\
\text { up to rounding) } \\
x_{1}=2 x_{2}>0\end{array}$ & $\begin{array}{c}\text { efficiency- } \\
\text { guided }\end{array}$ \\
\hline$Z$ & $\begin{array}{c}x_{1}=x_{2}=0 \\
x_{1}=x_{2}>0\end{array}$ & 7 & 4 & $2 x_{2}>x_{1}>x_{2}>0$ & 3 & 6 \\
& $\%$ & $33 \%$ & $19 \%$ & 1 & $14 \%$ & $29 \%$ \\
\hline
\end{tabular}

Table 2: Types of conditional donations in the two-sided treatment

The fact that in the one-sided treatment recipients in their imaginary role as dictators are less generous than actual dictators, supports the view that the lack of generosity in the 
two-sided treatment may be triggered by pessimistic expectations. Likewise, more optimistic expectations may induce the opposite behavior. This discussion provides some clue why we do observe

Regularity 7: The possibility of two-sided contributions facilitates the emergence of more diverse behavioral types, especially by making egoistic (or distrustful) and efficiency-minded (or trustful) behavior more prominent.

In our view, the striking features of the two-sided as opposed to the one-sided gift relationship, as stated by Regularities 6 and 7, are due to the fact that for mutual gifts there is no purely distributional fairness constraint. All gift levels $x_{1}, x_{2}$ with $0 \leq x_{1}, x_{2} \leq 10$ can be justified as fair if the partner is expected to be either equally generous or equally egoistic. In sum,

General conclusion 8: People engage in gift giving even when there is only one-sided exchange and even when there are no efficiency gains. The level of giving is bounded (from above) by fairness constraints in the sense that gifts do not put gift givers at a disadvantage. Efficiency gains further gift giving when efficient outcomes are expected. This leads to much more behavioral heterogeneity in two-sided than in onesided gift-relations.

\section{Conclusions}

Let us summarize the most relevant effects:

- Actual dictators never put themselves at a disadvantage, i.e. they always obey the fairness constraints. They also expect others to do so. An increase of $m$ from 1 to 2 induces lower sacrifices ${ }^{6}\left(x_{1}^{r}>x_{2}^{r}\right)$ but larger gifts $\left(x_{1}^{r}<2 x_{2}^{r}\right)$.

\footnotetext{
${ }^{6}$ A related observation has been made by Bolton and Ockenfels (2000b). They found that increasing efficiency in an ultimatum game by multiplying the payoff of the proposer for each possible outcome by a constant decreases the acceptance rate of a given offer. In other words, rejection rates are positively correlated with the associated efficiency loss if fairness dictates so.
} 
- Hypothetical dictators are slightly less generous. This seems mainly due to their pessimistic expectations concerning others' generosity. The striking difference in beliefs about others' behavior speaks for role dependent expectations.

- In mutual gift giving the fairness constraint (that the level of gifts can be justified by hopes which do not put the gift giver at a disadvantage) becomes non-binding. Every choice is justifiable as fair, given 'appropriate' beliefs about the behavior of the opponent. This reasonably explains the considerably larger heterogeneity of mutual gifts.

- Only in the one-sided treatment, efficiency gains inspire larger gifts but reduce sacrifices whereas in mutual gift giving also sacrifices increase with $m\left(x_{2}^{r}>x_{1}^{r}\right)$.

Our findings are in line with simple theories of fairness such as Fehr and Schmidt (1999) and Bolton and Ockenfels (2000a). However, if furthering efficiency is very cheap two other studies found more evidence for efficiency considerations. Charness and Rabin (2000) and Engelmann and Strobel (2000) found evidence that if furthering efficiency is free of any monetary costs, some subjects choose efficient outcomes even if this puts the individual so deciding at a disadvantage. Charness and Rabin (2000) in addition found some evidence for efficiency considerations if the efficient choice is costly, but much cheaper than in our experiment. The fact, however, that one rarely observes unconditional cooperation in mutual gift giving games such as prisoner' dilemma games and our comparison of one-sided and two-sided giving suggest that fairness often dominates efficiency considerations. 


\section{References}

Bolton, G.E., Katok, E. and Zwick, R. (1998). "Dictator Game Giving: Rules of Fairness versus Acts of Kindness." International Journal of Game Theory, 27, 269-299.

Bolton, G.E. and Ockenfels, A. (2000a). "ERC: A Theory of Equity, Reciprocity and Competition." American Economic Review, March 2000, 90(1), 166-93.

Bolton, G.E. and Ockenfels, A. (2000b). "A Stress Test of Fairness Measures in Models of Social Utility," Working Paper, University of Magdeburg.

Charness, G. and Rabin, M. (2000). "Social Preferences: Simple Tests and a New Model," working paper, Berkelee.

Engelmann, D. and Strobel, M. (2000). “An Experimental Comparison of the models of Bolton and Ockenfels and Fehr and Schmidt," working paper, Humboldt University.

Fehr, E., and Schmidt, K. (1999). "A Theory of Fairness, Competition, and Cooperation," Quarterly Journal of Economics, 114, 817-868.

Festinger, L. (1964). Conflict, Decision, and Dissonance. Stanford: Stanford University Press.

Hobbes, Th. (1998). On the Citizen. (Cambridge Texts in the History of Political Thought) Cambridge: Cambridge University Press.

Ng, Yew-Kwang (1979): Welfare Economics. London and Basingstoke: Macmillan.

Ockenfels, A. (1999). "Fairness, Reziprozitaet und Eigennutz." Tuebingen: Mohr Siebeck.

Selten, R. and Ockenfels, A. (1998). “An Experimental Solidarity Game,” Journal of Economic Behavior and Organization, 34(4), 517-539.

Shapiro, E.G. (1975). "Effects of Future Interaction in Reward Allocation Dyads: Equity or Equality," Journal of Personality and Social Psychology, 31, 873-880. 


\section{Appendix}

The following list presents first 24 independent observations for the two-sided treatment, then the 24 choices of the $X$-participants, and finally the 24 choices of the $Y$-participants in the one-sided treatment. After the participant number, a " 1 " in the second column indicates that both control questions were answered correctly; "0" signals at least one mistake. The third column gives the choice vectors $\left(x_{1}, x_{2}\right)$, i.e. the gift $x_{1}$ in case of $m=$ 1 and $x_{2}$ for $m=2$, where the last 24 choice vectors (numbers 49 to 72) are, of course, purely hypothetical. The final column lists the expectations concerning the average gift giving by others, both for $m=1$ and $m=2$.

\begin{tabular}{|c|c|c|c|c|c|}
\hline \multirow[t]{2}{*}{ Player \# } & \multirow{2}{*}{$\begin{array}{c}\text { control } \\
\text { questions } \\
(1=\text { correct })\end{array}$} & \multicolumn{2}{|c|}{ Decisions } & \multicolumn{2}{|c|}{ Expectations } \\
\hline & & $x_{1}$ & $x_{2}$ & $x_{1}$ & $x_{2}$ \\
\hline \multicolumn{6}{|c|}{ Mutual gift giving } \\
\hline 1 & 1 & 0,00 & 10,00 & 0,00 & 10,00 \\
\hline 2 & 1 & 5,00 & 5,00 & 2,00 & 2,00 \\
\hline 3 & 0 & 2,50 & 2,00 & 1,50 & 1,80 \\
\hline 4 & 1 & 2,00 & 1,00 & 2,00 & 1,00 \\
\hline 5 & 1 & 3,00 & 5,00 & 2,00 & 4,00 \\
\hline 6 & 1 & 2,50 & 1,25 & 5,00 & 2,50 \\
\hline 7 & 1 & 4,00 & 2,00 & 3,00 & 1,50 \\
\hline 8 & 1 & 2,00 & 7,00 & 1,00 & 5,00 \\
\hline 9 & 1 & 0,00 & 0,00 & 1,00 & 1,00 \\
\hline 10 & 1 & 0,00 & 0,00 & 0,00 & 0,00 \\
\hline 11 & 0 & 10,00 & 5,00 & 0,00 & 0,00 \\
\hline 12 & 1 & 10,00 & 10,00 & 0,00 & 0,00 \\
\hline 13 & 1 & 4,00 & 4,00 & 4,00 & 4,00 \\
\hline 14 & 1 & 0,00 & 0,00 & 2,00 & 1,00 \\
\hline 15 & 1 & 0,00 & 0,00 & 0,00 & 2,00 \\
\hline 16 & 1 & 2,00 & 2,00 & 1,00 & 1,00 \\
\hline 17 & 0 & 5,00 & 3,00 & 5,00 & 1,00 \\
\hline 18 & 1 & 6,00 & 4,00 & 3,00 & 2,00 \\
\hline 19 & 1 & 4,00 & 8,00 & 4,00 & 4,00 \\
\hline 20 & 1 & 4,00 & 9,00 & 5,00 & 10,00 \\
\hline 21 & 1 & 0,00 & 1,00 & 0,00 & 0,00 \\
\hline 22 & 1 & 0,00 & 0,00 & 0,50 & 1,00 \\
\hline 23 & 1 & 0,00 & 0,00 & 1,00 & 1,00 \\
\hline 24 & 1 & 0,00 & 0,00 & 1,00 & 1,00 \\
\hline \multicolumn{6}{|c|}{ Dictator giving } \\
\hline 25 & 1 & 3,75 & 2,50 & 3,50 & 2,50 \\
\hline 26 & 1 & 5,00 & 3,00 & 4,00 & 3,00 \\
\hline
\end{tabular}




\begin{tabular}{|c|c|c|c|c|c|}
\hline & & & & & \\
\hline 27 & 1 & 2,50 & 1,50 & 1,50 & 0,50 \\
\hline 28 & 1 & 2,00 & 2,00 & 2,00 & 1,00 \\
\hline 29 & 1 & 2,50 & 2,50 & 4,00 & 4,00 \\
\hline 30 & 1 & 2,00 & 2,00 & 4,00 & 3,00 \\
\hline 31 & 1 & 5,00 & 2,50 & 4,00 & 2,00 \\
\hline 32 & 1 & 4,00 & 3,00 & 3,00 & 2,00 \\
\hline 33 & 1 & 1,00 & 1,00 & 2,00 & 1,00 \\
\hline 34 & 1 & 2,00 & 1,00 & 4,00 & 2,00 \\
\hline 35 & 1 & 2,00 & 1,00 & 4,00 & 2,00 \\
\hline 36 & 1 & 5,00 & 2,50 & 5,00 & 2,50 \\
\hline 37 & 0 & 10,00 & 5,00 & 10,00 & 5,00 \\
\hline 38 & 1 & 2,00 & 1,00 & 2,00 & 1,00 \\
\hline 39 & 1 & 1,00 & 1,00 & 1,00 & 1,00 \\
\hline 40 & 1 & 3,00 & 2,00 & 4,00 & 2,00 \\
\hline 41 & 1 & 0,00 & 0,00 & 0,00 & 0,00 \\
\hline 42 & 1 & 0,00 & 0,00 & 0,00 & 0,00 \\
\hline 43 & 1 & 2,00 & 1,00 & 2,00 & 1,00 \\
\hline 44 & 1 & 5,00 & 2,50 & 3,00 & 2,00 \\
\hline 45 & 1 & 5,00 & 3,00 & 3,00 & 2,00 \\
\hline 46 & 1 & 1,00 & 1,00 & 2,00 & 1,00 \\
\hline 47 & 1 & 2,00 & 1,00 & 1,00 & 1,00 \\
\hline 48 & 1 & 5,00 & 2,00 & 3,00 & 2,00 \\
\hline \multicolumn{6}{|c|}{ Recipients' hypothetical givings } \\
\hline 49 & 1 & 3,00 & \begin{tabular}{|l|}
2,00 \\
\end{tabular} & 2,00 & 1,00 \\
\hline 50 & 1 & 0,00 & 0,00 & 1,00 & 1,00 \\
\hline 51 & 1 & 1,00 & 1,00 & 1,00 & 1,00 \\
\hline 52 & 1 & 5,00 & 3,00 & 1,00 & 1,00 \\
\hline 53 & 1 & 0,00 & 0,00 & 0,00 & 0,00 \\
\hline 54 & 1 & 0,00 & 0,00 & 0,00 & 0,00 \\
\hline 55 & 1 & 10,00 & 10,00 & 0,25 & 0,25 \\
\hline 56 & 1 & 4,00 & 2,00 & 4,00 & 2,00 \\
\hline 57 & 1 & 2,00 & 1,00 & 2,00 & 1,00 \\
\hline 58 & 1 & 2,00 & 1,00 & 2,00 & 1,00 \\
\hline 59 & 1 & 2,00 & 1,00 & 2,00 & 2,00 \\
\hline 60 & 1 & 3,00 & 3,00 & 3,00 & 3,00 \\
\hline 61 & 1 & 2,00 & 2,00 & 2,00 & 2,00 \\
\hline 62 & 1 & 5,00 & 2,50 & 0,00 & 0,00 \\
\hline 63 & 1 & 0,00 & 0,00 & 5,00 & 2,50 \\
\hline 64 & 1 & 0,00 & 0,00 & 0,00 & 0,00 \\
\hline 65 & 1 & 0,00 & 0,00 & 0,00 & 0,00 \\
\hline 66 & 1 & 2,00 & 1,00 & 0,00 & 0,00 \\
\hline 67 & 1 & 1,00 & 2,00 & 2,00 & 4,00 \\
\hline 68 & 1 & 5,00 & 3,00 & 0,00 & 0,00 \\
\hline 69 & 0 & 5,00 & 5,00 & 2,00 & 2,00 \\
\hline 70 & 0 & 3,00 & 1,50 & 3,00 & 1,50 \\
\hline 71 & 1 & 0,00 & 0,00 & 2,00 & 1,00 \\
\hline 72 & 1 & 0,50 & 0,50 & 1,00 & 2,00 \\
\hline
\end{tabular}

